\title{
A NOTE ON THE SECRETARY PROBLEM
}

\section{R. BARTOSZYŃSKI}

Institute of Mathematics, Polish Academy of Sciences, Warszawa, Poland

and

\section{Z. GOVINDARAJULU}

Department of Statistics, University of Kentucky, Lexington, Kentucky, U.S.A.

\section{Introduction}

In this note we shall consider a version of the so-called secretary problem (see, for instance, Gilbert and Mosteller, 1966) when the objective is to find one of the three best candidates. To make this note self-contained, we state briefly the secretary problem.

Let $x_{1}, \ldots, x_{n}$ be the random permutation of numbers $1, \ldots, n$ (the "ranks" of candidates), and let

$$
y_{i}=\text { number of indices } j \leqslant i \text { for which } x_{j} \leqslant x_{i} .
$$

Thus, $y_{i}$ is the relative rank of $x_{i}$ among the first $i$ terms. We assume that $y_{1}, y_{2}, \ldots$ are observed sequentially while $x_{1}, x_{2}, \ldots$ are not observable.

After observing $y_{1}, \ldots, y_{i}(i<n)$ we may decide to stop, or to continue observations. For $i=n$ we must stop. If we stop at time $i$, we receive $a, b, c$ or nothing $(a \geqslant b \geqslant c \geqslant 0)$ depending on whether $x_{i}$ (the "true" rank of stopped candidate) is $1,2,3$ or $>3$. The problem is to find the optimal strategy of stopping and the expected return under this strategy.

Using the general results in theory of optimal stopping, one can show that the optimal strategy is of the form: stop at first $k$ for which either of the following conditions holds:
(a) $y_{k}=1$ and $k \geqslant r$,
(b) $y_{k}=2$ and $k \geqslant s$,
(c) $y_{k}=3$ and $k \geqslant t$,
(d) $k=n$,

where $1<r<s \leqslant t \leqslant n$. 
Thus, times $1, \ldots, r-1$ form an "orientation period" $A$ when we observe $y_{1}, \ldots, y_{r-1}$ without stopping, followed by three periods, $B=\{r, \ldots, s-1\}$, $C=\{s, \ldots, t-1\}$ and $D=\{t, \ldots, n\}$ with the property that we stop at first occasion when relative rank 1 occurs in $B$, or relative rank 1 or 2 occurs in $C$, or relative rank 1,2 or 3 occurs in $D$.

We shall find the asymptotic values for optimal "thresholds" $r, s$, and $t$. Though the results are of little (if any) general interest, they possess some curiosity value, since the numerical evaluations exhibit certain rather unexpected properties of variability of $r, s$, and $t$ as one changes the incentives $a, b$ and $c$.

\section{Exact form of expected return}

One can show that the expected return for the stopping rule based on numbers $1<r<s \leqslant t \leqslant n$ equals:

$$
\begin{aligned}
& \text { (1) } E(r, s, t)=\frac{a+b+c}{n(n-1)(n-2)}\left[(r-1) \sum_{k=r}^{s-1} \frac{(n-k)(n-k-1)}{k-1}+\right. \\
& +(r-1)(s-2) \sum_{k=s}^{t-1} \frac{(n-k)(n-k-1)}{(k-1)(k-2)}+ \\
& +(r-1)(s-2)(t-3) \sum_{k=t}^{n} \frac{(n-k)(n-k-1)}{(k-1)(k-2)(k-3)}+ \\
& +(r-1)(s-r)(t-3) \sum_{k=t}^{n} \frac{1}{k-3}+2(r-1)(s-2) \sum_{k=s}^{t-1} \frac{n-k}{k-2}+ \\
& +2(r-1)(s-2)(t-3) \sum_{k=t}^{n} \frac{n-k}{(k-2)(k-3)}+ \\
& \left.+(r-1)(r-2)(t-3) \sum_{k=1}^{n} \frac{1}{k-3}\right]+ \\
& +\frac{a}{n(n-1)(n-2)}\left[2(r-1) \sum_{k=r}^{s-1}(n-k)+(r-1) \sum_{k=r}^{s-1}(t-1-k)+(r-1)(r-2)(t-r)\right]+ \\
& +\frac{b}{n(n-1)(n-2)}\left[(r-1)(s-r)(t-s)+(r-1) \sum_{k=r}^{s-1}(n-k)+(r-1)(r-2)(t-s)\right] .
\end{aligned}
$$

We shall not give the proof in all details; instead, we shall illustrate the method of deriving (1) by considering some selected special cases.
Let $i_{1}, i_{2}$, and $i_{3}$ be the (random) indices of places at which the true ranks 1,2 , and 3 appear in the permutation $x_{1}, \ldots, x_{n}$. Various terms in (1) correspond to different configurations of appearances of $i_{1}, i_{2}$, and $i_{3}$ in sets $A, B, C$, and $D$. Let us, for instance, consider the case $i_{1} \in D, i_{1}<i_{2}, i_{1}<i_{3}$. The probability that $i_{1}=k$ and $i_{2}, i_{3}>k$ equals

$$
\frac{1}{n} \cdot \frac{n-k}{n-1} \cdot \frac{n-k-1}{n-2}
$$

Since $i_{1}=k$ implies $y_{k}=1$, in the above case we stop at $k$ (and win a prize $a$ ) if we do not stop before $k$ (in which case we do not win anything, since $i_{2}>k$ and $i_{3}>k$ ). In order that we do not stop before $k$ (for $k \in D$ ), it is necessary that in the sequence $y_{1}, \ldots, y_{k-1}$ of relative ranks there is

(a) no term equal 1 at places $j=r, \ldots, k-1$;

(b) no term equal 2 at places $j=s, \ldots, k-1$;

(c) no term equal 3 at places $j=t, \ldots, k-1$.

Thus, if $x^{\prime}, x^{\prime \prime}$, and $x^{\prime \prime \prime}$ are three least elements among $x_{1}, \ldots, x_{k-1}$, then $x^{\prime}$ must appear at a place $j$ with $j \leqslant r-1, x^{\prime \prime}$ must appear at a place $j$ with $j \leqslant s-1$, and $x^{\prime \prime \prime}$ must appear at a place $j$ with $j \leqslant t-1$. The probability of such an event equals

$$
\frac{r-1}{k-1} \cdot \frac{s-2}{k-2} \cdot \frac{t-3}{k-3}
$$

Summing over all $k=t, \ldots, n$, we obtain the contribution to the expected return

$$
a \sum_{k=t}^{n} \frac{(r-1)(s-2)(t-3)(n-k)(n-k-1)}{n(n-1)(n-2)(k-1)(k-2)(k-3)}
$$

which accounts for the third sum in (1). In this case we may interchange $i_{1}, i_{2}$, and $i_{3}$, obtaining the same expression for probability of winning. Such symmetry is not always present, as may be illustrated by the following cases (which explain why it appears hopeless to try this method in the general case when we want to stop any of the best $m$ candidates).

Let us consider the case $i_{1}<i_{2}<i_{3}$ with $i_{1} \in A, i_{3} \in B$. Certainly, we do not stop at $i_{1}$ or $i_{3}$, and we could win only if we stop at $i_{2}$. Now, for $k>i_{3}$ we have $y_{k}>1$, and the only element appearing at places $k>i_{3}$ with relative rank 2 will be that at place $i_{2}$, while for $k>i_{2}$ we have $y_{k}>3$ and no stopping is possible. Thus, we lose if $i_{2} \in B$ (since in the set $B$ we stop only at relative rank 1), we win if $i_{2} \in C$, while for $i_{2}=k \in D$ we win only if no element with relative rank 3 appears at places $t, t+1, \ldots, k-1$. Since in all three cases stopping is at true rank 2 , we get the contribution to $E(r, s, t)$ equal

$$
b\left[\frac{(r-1)(s-r)(t-s)}{n(n-1)(n-2)}+\frac{(r-1)(s-r)(t-3)}{n(n-1)(n-2)} \sum_{k=t}^{n} \frac{1}{k-3}\right]
$$


Consider now the case $i_{2}<i_{3}<i_{1}$ with $i_{2} \in A, i_{3} \in B$. As distinct from the preceding case, here we win if $i_{1} \in B$ or $i_{1} \in C$, while for $i_{1}=k \in D$ the situation is the same as before. Since now we win by stopping the best candidate, the contribution to $E(r, s, t)$ equals

$$
a\left[\frac{r-1}{n(n-1)(n-2)} \sum_{k=r}^{s-1}(t-1-k)+\frac{(r-1)(s-r)(t-3)}{n(n-1)(n-2)} \sum_{k=t}^{n} \frac{1}{k-3}\right] .
$$

\section{Asymptotic solution}

Formula (1) permits to obtain the asymptotic form of the optimal stopping policy. Let $x \sim r / n, y \sim s / n$ and $z \sim t / n$. After some elementary calculations, one obtains

(2) $E(x, y, z)=(a+b+c)\left[x+2 x^{2}-\frac{1}{2} x^{3}-2 x y+\right.$

$$
\begin{gathered}
\left.-\frac{1}{2} \frac{x y}{z}+\frac{3}{2} x y^{2}+x \log (y / x)-\frac{3}{2} x y z\right]+ \\
+a\left[2 x y-2 x^{2}+\frac{1}{2} x^{3}-\frac{3}{2} x y^{2}+x y z\right]+b\left[x y z-\frac{3}{2} x y^{2}-x^{2}+x y+\frac{1}{2} x^{3}\right] .
\end{gathered}
$$

In order to be able to compare the expected returns, let us put $a+b+c=1$; since $a \geqslant b \geqslant c \geqslant 0$, we have then $0 \leqslant b \leqslant 1 / 2$ and $0 \leqslant c \leqslant \min (b, 1-2 b)$.

Differentiating $E(x, y, z)$ with respect to its arguments and solving the resulting equations, we obtain

(3) $z=(1+2 c)^{-1 / 2}$

$$
y= \begin{cases}\frac{b+2 c+(1+2 c)^{1 / 2}-\left\{1-10 c+(b+2 c)\left[b+2 c+2(1+2 c)^{1 / 2}\right]\right\}^{1 / 2}}{6 c} & \\ (1+b)^{-1} & \text { if } c \neq 0 \\ & \text { if } c=0,\end{cases}
$$

while $x$ is equal to the root of the equation

$$
3 c x^{2}-4(b+2 c) x+2 \log x=2 \log y-y\left(b+2 c+z^{-1}\right)-1
$$

with $y$ and $z$ given by (4) and (3).

Observe that if $b=c=0$, we get $y=z=1$, and $x=e^{-1}$, which is the wellknown solution of the secretary problem with the prize given only for getting the best candidate. For $a=b=1 / 2, c=0$ we get $z=1, y=2 / 3$, and $x$ given by the equation $\frac{3}{2} x=e^{x-1}$, which again is the solution given by Gilbert and Mosteller (1966).

\section{Numerical values}

The table below gives some numerical values for the optimal $x, y$ and $z$, and the expected return $E(x, y, z)$ under the optimal policy. It is worth observing that the value $x$ changes very little (see Fig. 1 showing changes for $b=0.3$ and $0 \leqslant c \leqslant 0.3$ ). The reason for such small changes is, qualitatively speaking, quite clear: an increase of $x$ increases the risk of missing all "prized" candidates altogether, and hence making no choice at all, while the decrease of $x$ enhances the risk of getting too high value with lowest relative rank so far, thus increasing the risk of making a wrong choice.

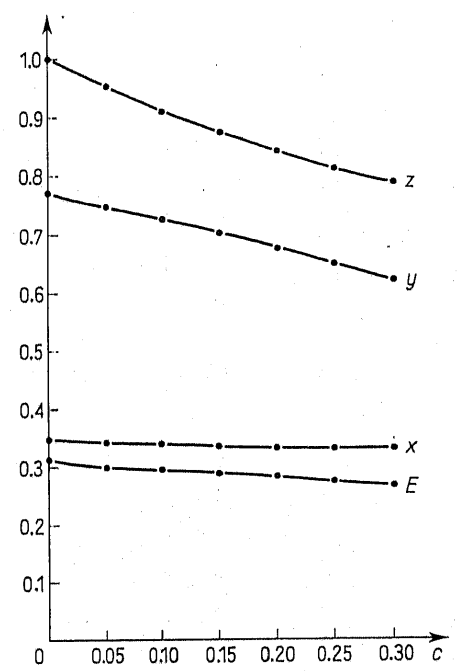

Fig. 1. Optimal values $x, y, z$ and $E$ for $b=0.3$ and $0 \leqslant c \leqslant 0.3$

The initial "orientation period", determined by the value of $x$, is maximal when $b=c=0$, and tends to shorten as the prize is split among the three best candidates. The minimum, however, is not attained for even split, i.e. when $a=b$ $=c=1 / 3$. In fact, for fixed $b, x$ decreases with the increase of $c$, while for fixed $c$, as $b$ increases from its minimal to maximal value, $x$ first decreases and then starts to increase (the latter changes, however, occur only in the third and fourth decimals).

This means that for a fixed prize for the third-in-rank candidate, the orientation period is shortest when the prize for the second best candidate lies in between its minimal and maximal value. 
Finally, lest us observe that splitting the prize has always a detrimental effect on the expected return. When $a=1, b=c=0$ (i.e. if we win a unit amount only for getting the best candidate), the expected return is $e^{-1}=0.3679$. When we get $1 / 3$ for stopping at any of the three top candidates, the expected return is lowest, and equals only 0.2616 .

Table 1

Optimal thresholds $x, y$, and $z$, and the optimal return $E$ for different prizes $b$ and $c$

\begin{tabular}{cl|cccc}
\hline$c$ & $b$ & $x$ & $y$ & $z$ & $E$ \\
\hline 0 & 0 & 0.3679 & 1.0000 & 1.0000 & 0.3679 \\
& 0.1 & 0.3594 & 0.9091 & 1.0000 & 0.3464 \\
& 0.2 & 0.3531 & 0.8333 & 1.0000 & 0.3281 \\
& 0.3 & 0.3489 & 0.7692 & 1.0000 & 0.3124 \\
& 0.4 & 0.3468 & 0.7143 & 1.0000 & 0.2987 \\
& 0.5 & 0.3470 & 0.6667 & 1.0000 & 0.2868 \\
0.1 & 0.1 & 0.3507 & 0.8849 & 0.9129 & 0.3323 \\
& 0.2 & 0.3445 & 0.7956 & 0.9129 & 0.3136 \\
& 0.3 & 0.3410 & 0.7258 & 0.9129 & 0.2983 \\
& 0.4 & 0.3402 & 0.6689 & 0.9129 & 0.2851 \\
& 0.45 & 0.3409 & 0.6442 & 0.9129 & 0.2793 \\
0.2 & 0.2 & 0.3379 & 0.7501 & 0.8452 & 0.2985 \\
& 0.3 & 0.3358 & 0.6771 & 0.8452 & 0.2774 \\
& 0.4 & 0.3373 & 0.6208 & 0.8452 & 0.2717 \\
0.3 & 0.3 & 0.3342 & 0.6236 & 0.7906 & 0.2696 \\
& 0.35 & 0.3364 & 0.5957 & 0.7906 & 0.2640 \\
& & & & & \\
0.33 & 0.33 & 0.3367 & 0.5868 & 0.7746 & 0.2616 \\
\hline
\end{tabular}

\section{Reference}

J. G i l be r t and F. Most elle r, Recognizing the maximum of a sequence, Amer. Stat. Assoc. 61 (1966), pp. $35-73$.
Presented to the semester MATHEMATICAL STATISTICS September 15-December 18, 1976

\section{REDUCIBICITY OF STATISTICAL STRUCTURES AND DECISION PROBLEMS}

\section{R. BARTOSZYŃSKI and E. PLESZCZYŃSKA}

Institute of Mathematics, Polish Academy of Sciences, Warszawa, Poland, Institute of Computer Science, Polish Academy of Sciences, Warszawa, Poland

\section{Introduction}

The object of this paper is to analyze the necessary conditions which must be met in order that the decision procedures applicable to one statistical decision problem could also be used in other problems obtained from the original by suitable transformations. The basic concept is that of $\mathscr{F}$-reducibility of two statistical structures (and of two statistical decision problems), where $\mathscr{F}$ is a family of mappings of the underlying sample spaces. The paper gives conditions under which proximity of measures in one structure is preserved in the reduced structure (which is a prerequisite for robustness of procedures), and under which a parameter in one structure can also serve as a parameter in the other.

Moreover, the conditions are given under which certain desirable properties of parameters are preserved in the reduced structure. Applicability of the introduced concepts to statistical analysis of stochastic processes is discussed.

\section{Reducible statistical structures}

By a statistical structure we shall mean a triple $(\mathscr{X}, \mathscr{A}, \mathscr{P})$, where $\mathscr{X}$ is an arbitrary set, $\mathscr{A}$ is a $\sigma$-field of subsets of $\mathscr{X}$, and $\mathscr{P}$ is a family of probability measures on $(\mathscr{X}, \mathscr{A})$.

Let now $(\mathscr{X}, \mathscr{A})$ and $\left(\mathscr{X}^{\prime}, \mathscr{A}^{\prime}\right)$ be two measurable spaces, and let $\mathscr{K}$ and $\mathscr{K}^{\prime}$ denote respectively the classes of all probability measures on $(\mathscr{X}, \mathscr{A})$ and $\left(\mathscr{X}^{\prime}, \mathscr{A}^{\prime}\right)$. Next, let $\mathscr{F}$ be a class of $\mathscr{A}-\mathscr{A}^{\prime}$ measurable mappings $f: \mathscr{X} \rightarrow \mathscr{X}^{\prime}$. With each $f \in \mathscr{F}$ we can connect a mapping $\bar{f}: \mathscr{K} \rightarrow \mathscr{K}^{\prime}$ by putting

$$
\overline{f P}\left(A^{\prime}\right)=P\left(f^{-1}\left(A^{\prime}\right)\right), \quad A^{\prime} \in \mathscr{A}^{\prime} .
$$

Let $\bar{f}^{-1} P^{\prime}=\left\{P: \overline{f P}=P^{\prime}\right\}$. Futhermore, for $\mathscr{P} \subset \mathscr{K}$ and $\mathscr{P}^{\prime} \subset \mathscr{K}^{\prime}$, let $\overline{\mathscr{F}}(\mathscr{P})=\bigcup_{f \in \mathscr{F}}\{\bar{f}(\mathscr{P})\}=\left\{P^{\prime} \in \mathscr{K}^{\prime}: \bar{f} P=P^{\prime}\right.$ for some $P \in \mathscr{P}$ and some $\left.f \in \mathscr{F}\right\}$, 